\title{
THE POSSIBLE ROLE OF RADIATIVE ACCELERATION IN SUPPORTING EXTENDED ATMOSPHERES IN BE STARS
}

\author{
R. L. KURUCZ and R. E. SCHILD \\ Center for Astrophysics, Harvard College Observatory, and Smithsonian Astrophysical Observatory \\ Cambridge, Mass., U.S.A.
}

\begin{abstract}
A detailed calculation of the radiative acceleration in B-type stars shows it to be a doublepeaked function of effective temperature at small optical depths. The two peaks are shown to coincide approximately with peaks in the distribution of mean $\mathrm{H} \alpha$ emission strength as a function of $B-V$ color in $\mathrm{Be}$ stars. These facts suggest that radiation may play an important role in the support of the Be star extended atmosphere.
\end{abstract}

\section{Introduction}

Whereas the earliest papers on the Be stars stressed the role of rotation (Struve, 1945; Slettebak, 1949), more recent work has tended to stress the role of radiation processes. The most recent paper by Marlborough and Zamir (1975) suggests that stellar winds may be important in maintaining a mass of gas at distances of several stellar radii above the rotationally distorted stars. Thus our conception of $\mathrm{Be}$ stars as rapidly rotating stars in which centrifugal force maintains a shell of gas several stellar radii above the photosphere, is slowly giving way to a picture wherein rotation effectively reduces the surface gravity to the point where the radiation field generates a stellar wind, which constantly replenishes the shell which is in turn constantly expanding away from the star.

We wish to further substantiate this view with results of model atmosphere calculations and a comparison with data for normal Be stars. Schild and Romanishin (1975) have recently analysed the data for $41 \mathrm{Be}$ stars in clusters and shown that the strength of $\mathrm{H} \alpha$ emission is a double peaked function of intrinsic $(B-V)$ color, with peaks near $(B-V)=-0.28$ and $(B-V)=-0.12$ corresponding to spectral types $B 1$ and B8. We are not aware of any explanation of these peaks in terms of strictly rotational effects in the post-main-sequence evolution of stars. In this paper we present results of model atomosphere calculations which may explain the double peak in terms of an effect of radiative acceleration.

\section{Calculations}

Kurucz (1975) has recently completed a new model atmosphere grid covering the range $5500 \mathrm{~K}$ to $50000 \mathrm{~K}$. This grid is a revision and extension of the work by Kurucz et al. (1975) on early type stars. The new models have an improved treatment of line opacity and essentially encompass the range of temperatures for models that can be computed without including molecular lines. In the new calculations the line 
list computed by Kurucz and Peytremann (1975) was edited down to fewer than 1000000 lines and the statistical distribution function representation of the line opacity was tabulated at many additional temperature values. Wavelength bandpasses were reduced from $100 \AA$ to $25 \AA$ in the visible to allow calculation of intermediate band colors.

These new models seem to be good representations of real stars. The model for Vega is in excellent agreement with the new calibration of Hayes and Latham (1975) and reproduces the Balmer profiles of Peterson (1968). The $U B V$ and uvby colors predicted from these models are normalized so that the model for Vega has the colors of Vega. Kurucz is currently checking these models further by comparing detailed spectral calculations with satellite and ground-based observations.

The limitations on these models that affect the discussion here are several. First, these are plane-parallel models for non-rotating stars. Rotating the models in the manner of Collins (1966) or Maeder and Peytremann (1972) would change the colors somewhat. Second, the models are not numerically reliable above $\tau_{\text {Ross }}=10^{-4}$ because the surface was set at $3 \times 10^{-5}$ or $10^{-5}$. The calculation should extend another decade or two to investigate the $\mathrm{H} \alpha$ core. Third, at these shallow optical depths, non-LTE becomes important. Nonetheless we feel that these models are adequate to make the qualitative argument presented here and are likely to be an improvement over the models used by Lucy and Solomon (1970) in their discussion of radiative effects.

Radiative acceleration is determined by the integral

$$
a=\frac{4 \pi}{c} \int \kappa_{\nu} H_{\nu} \mathrm{d} \nu
$$

where $\kappa_{\nu}$ is the total monochromatic mass absorption coefficient and $H_{\nu}$ is the flux. Since the total flux $\int H_{\nu} \mathrm{d} \nu$ is proportional to $T_{\text {eff }}^{4} a$ is roughly proportional to $T_{\text {eff }}^{4}$ However since $\kappa_{\nu}$ varies through the atmosphere, and with gravity and effective temperature, and since the radiation field is highly non-gray, we must work with a grid of models to predict where the observable effects might appear.

Since the Be effect appears first in the core of $\mathrm{H} \alpha$ let us look at a shallow layer $\tau_{\text {Ross }}=10^{-4}$ which would influence the $\mathrm{H} \alpha$ core. In Figure 1 we plot log radiative acceleration against $\log$ effective temperature for the new models of effective temperature $10000 \mathrm{~K}$ and hotter. The gravities range from 2 to 5 in steps of 0.5 . A line with a slope of 4 is indicated to show a proportional to $T_{\mathrm{eff}}^{4}$. Note that there are two peaks above this slope at $T_{\text {eff }} \sim 12000$ to $13000 \mathrm{~K}$ and $T_{\text {eff }} \sim 40000$ to $50000 \mathrm{~K}$ with a minimum in between. Note that the higher gravity models generally have higher accelerations because increased pressures lead to decreased ionization and increased opacity. At greater optical depths we find that the minimum disappears and that increasing opacity shifts the curves upward.

In Figure 2 we plot $\kappa_{\nu} H_{\nu}$ for three models to show the source of the minimum and maxima. The first maximum, at effective temperature $13000 \mathrm{~K}$ arises from absorption by singly ionized iron group lines in the region around $2600 \AA$. The second maximum, at $40000 \mathrm{~K}$, arises from the absorption lines of multiply ionized light elements spread throughout the far ultraviolet. The minimum reflects the lack of 


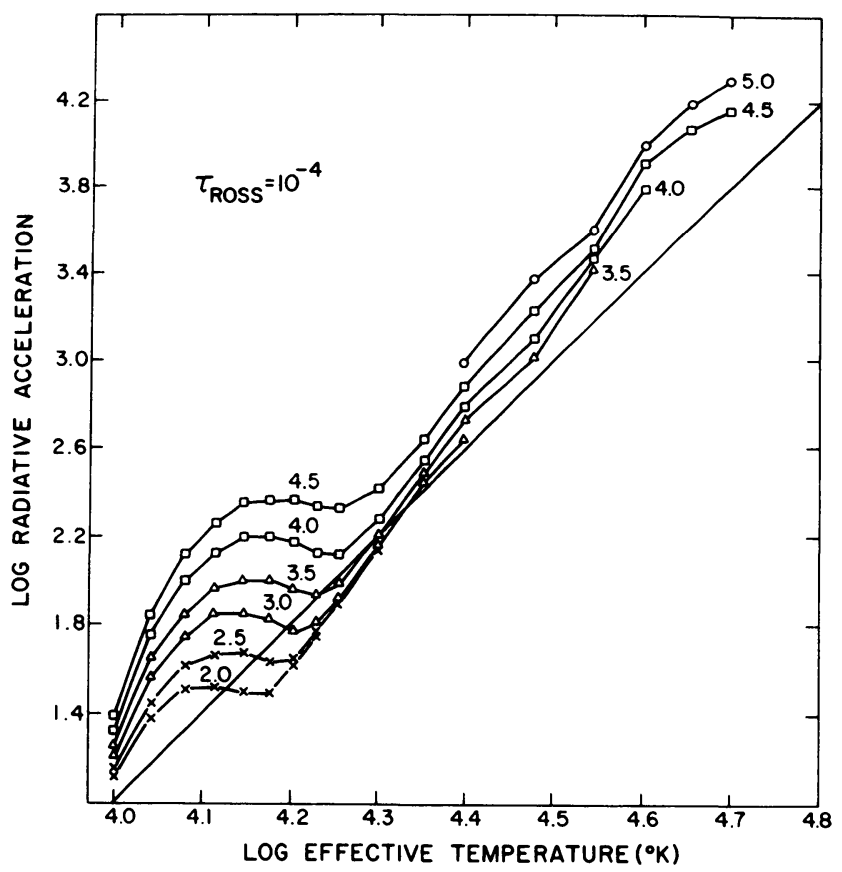

Fig. 1. Radiative acceleration is shown as a function of effective temperature for several choices of $\log g$ as shown for each curve. The straight line represents the factor proportional to $T_{\text {eff. }}^{4}$.

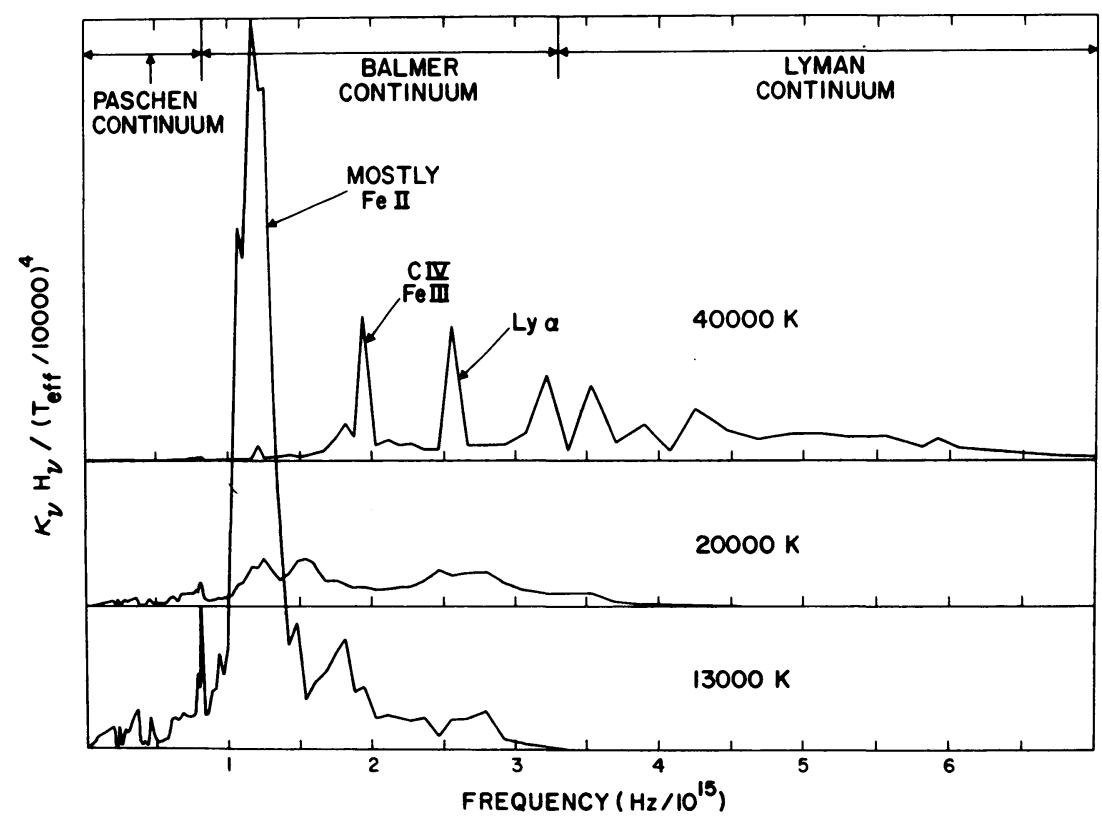

Fig. 2. The monochromatic term $\kappa_{\nu} H_{\nu}$ plotted as a function of frequency, to identify the contributors to the radiative acceleration. Note especially the large peak due to singly ionized iron group elements in the near ultraviolet for late B-type stars. 
suitable absorption lines at intermediate temperatures. A more detailed investigation could easily be performed (if computer time were available) by directly synthesizing the spectra and identifying individual lines.

In Figure 3 we replot the data from Figure 1 to show the maxima and minimum more clearly by removing the $T_{\text {eff }}^{4}$ dependence so the slope is nominally zero. Since

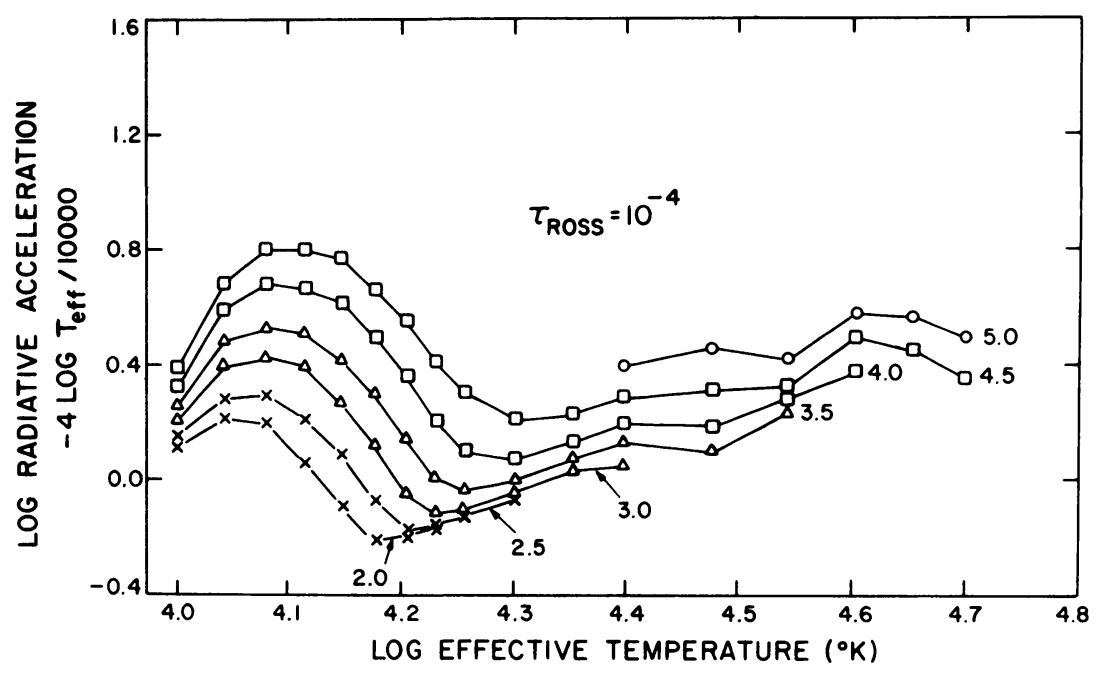

Fig. 3. Same results as in Figure 1, but the factor of $T_{\text {eff }}^{4}$ has been divided out to emphasize opacity effects.

the gravity at which a model becomes unstable to radiative acceleration, $g=a$ at any depth, roughly varies as $T_{\text {eff }}^{4}$, this figure approximately shows the stability of the outer layers as the model as a whole approaches instability.

In Figure 4 we show the results of Figure 2 transformed to the observational variable $(B-V)$ for a comparison with the measured $\mathrm{H} \alpha$ equivalent widths from Schild and Romanishin (1975). Both the theoretical and observational curves may have systematic errors. As noted earlier, the theoretical curves are affected by rotation; Collins (1966) has shown that rotating stars are redder than their nonrotating counterparts, by amounts that depend upon rotational velocity and aspect. Also, because the energy distributions of $\mathrm{Be}$ stars are generally distorted by continuum emission from gas above the stellar photosphere, the $(B-V)$ colors given by Schild and Romanishin are estimated from the colors of normal stars in the color magnitude diagrams of the clusters studied. Both of these effects may amount to errors of a few hundredths of a magnitude in $(B-V)$. We believe from the comparison in Figure 4 that the strength of $\mathrm{H} \alpha$ emission in post-main-sequence $\mathrm{Be}$ stars is correlated with $(B-V)_{0}$ color in about the same way as radiative acceleration. This suggests, but by no means proves, that the strength of the gas shell depends upon the strength of the radiative acceleration near the surface of the star.

Finally, we note a comparison with the work of Lucy and Solomon (1970). Their work on radiative acceleration took into account only the resonance lines of the light 


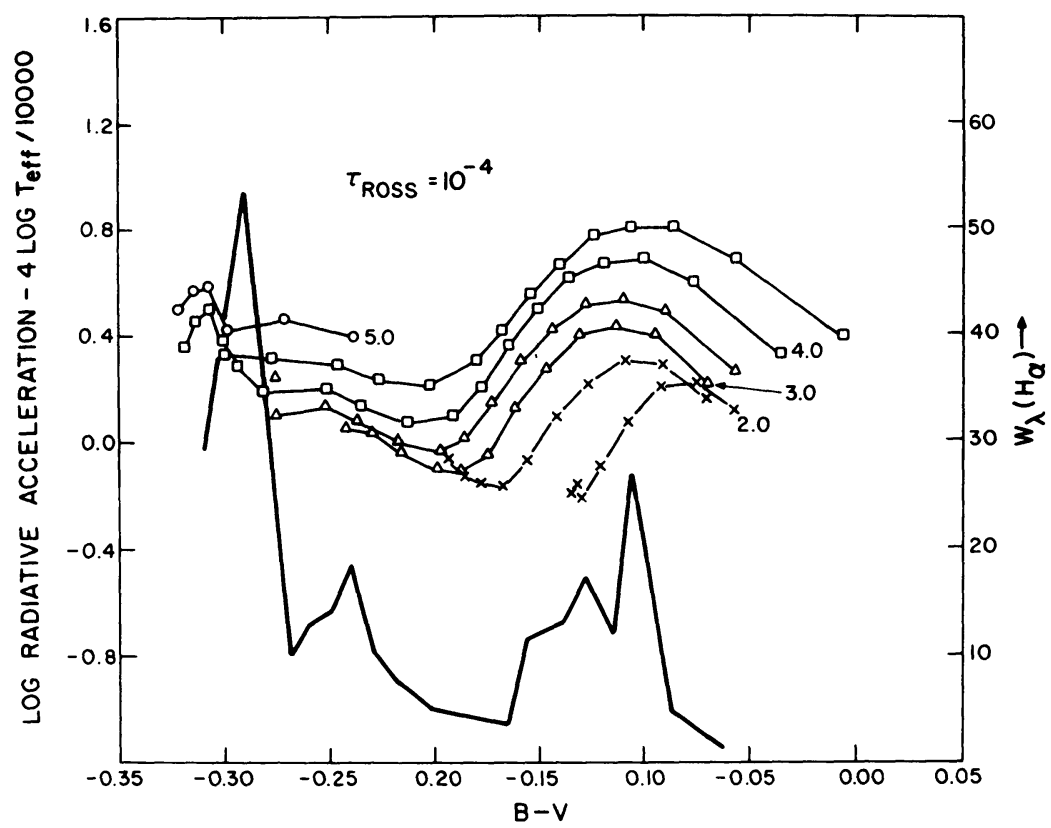

Fig. 4. Radiative acceleration as function of $(B-V)$ compared with data on $\mathrm{H} \alpha$ emission line equivalent width for Be stars in clusters.

metals, which are in the ultraviolet spectral region near the peak of the Planck function for the Be stars. As noted by Schild and Romanishin (1975), their results predict a secondary peak near the minimum of the $\mathrm{H} \alpha$ equivalent width curve. We attribute our $13000 \mathrm{~K}$ secondary peak to the inclusion of weaker lines in our calculations. It would be particularly desirable to compute a self-consistent $\mathrm{Be}$ star model which includes effects of radiation transfer and a detailed calculation of envelope support or a stellar wind, analogous to the self-consistent Of star model of Castor, Abbott, and Klein (1975).

\section{References}

Castor, J., Abbott, D., and Klein, R.: 1975, Astrophys. J. 195, 157.

Collins, G. W., II.: 1966, Astrophys. J. 146, 914.

Hayes, D. and Latham, D.: 1975, Astrophys. J. $197,593$.

Kurucz, R.: 1975, in preparation.

Kurucz, R. and Peytremann, E.: 1975, Smithsonian Astrophysical Observatory Special Report No. 362.

Kurucz, R., Peytremann, E., and Avrett, E.: 1975, Blanketed Model Atmospheres for Early-Type Stars (Washington: Smithsonian Institution Press), $189 \mathrm{pp}$.

Lucy, L. and Solomon, P.: 1970, Astrophys. J. 159, 879.

Maeder, A. and Peytremann, E.: 1972, Astron. Astrophys. 21, 279.

Marlborough, J. M. and Zamir, M.: 1975, Astrophys. J. 195, 145.

Peterson, D.: 1968, Smithsonian Astrophysical Observatory Special Report No. 293.

Schild, R. and Romanishin, W.: 1976, Astrophys. J. 204, 493.

Slettebak, A.: 1949, Astrophys. J. 110, 498.

Struve, O.: 1945, Pop. Astron. 53, 201, 259. 


\section{DISCUSSION}

Slettebak: Do you take gravity darkening into account in your computations?

Schild: No. In fact there has not been a calculation of a stellar model here. All this has been is Kurucz's calculations of the line opacity for normal stars.

Slettebak: If you believe the von Zeipel theorem or a variation thereof, a darkened equator would play a smaller role in supporting an extended Be star atmosphere.

Schild: We do expect the temperature gradient in Be stars to differ from that of a normal star, but we will not get to these questions until we have a self-consistent Be star model.

Hutchings: These Fe II lines below the Balmer limit: where are they, and are they observed?

Schild: I'm not sure if you expect them to be observed. As I recall, spectra at wavelengths near the atmospheric cutoff begin to show large numbers of iron lines, especially if you go to somewhat lower temperatures.

Snijders: In answer to Hutchings' question, I should like to point out that the results by various authors (mostly in press now) obtained with both the S-59 experiment and the Copernicus satellite show that the Fe II lines dominate the spectrum between $\lambda 2000$ and $\lambda 3000 \AA$ in late B-type stars, in good agreement with the Schild and Kurucz predictions. Further, Schild warned that his results are based on the LTE assumption and that this could be a serious source of errors. I should like to mention as yet unpublished non-LTE results for late B-type stars obtained by me which suggest that at $\tau_{\text {Rosseland }} \leqslant 10^{-4}$ the excited levels (when not metastable) are systematically underpopulated. This could considerably diminish the importance of radiation pressure from weak lines in late B-type stars.

Goldberg: Circumstellar shells were originally postulated because lines from metastable levels were found to be abnormally strong. Therefore, it's hard to understand how the excited levels can be populated in LTE. 